\title{
Resource Allocation Techniques for Cellular Networks in TV White Space Spectrum
}

\author{
Farzad Hessar and Sumit Roy Dept. of Electrical Engineering Department \\ University of Washington, Seattle, WA \\ Email: \{farzad, sroy\}@u.washington.edu
}

\begin{abstract}
TV white space (TVWS) represents unused TV channels - resulting from the transition to digital over-the-air broadcasting - made available by FCC for unlicensed secondary users. This presents opportunities for new wireless networks and services, for example as a complement to licensed 4G cellular networks as a mechanism for data offloading, in analogy with the use of 802.11 WLAN hotspot networks for current 3G networks. However, certain design challenges must be addressed - TVWS spectrum is characterized by significant (spatial) variability in number and quality (measured by signal to interference and noise ratio) of available channels. Therefore, channel allocation that seeks to optimize some overall network performance metric - must be adapted to this variation while complying with FCC regulations. In this paper, we explore the problem of resource allocation in TVWS spectrum by developing models for channel availability based on co-channel interferences among secondary users as well as primary-to-secondary interference. We define two formulations: a) based on maximizing number of allocated channels, and b) based on total network throughput. Techniques for solving these two formulations are explored and the results are compared through numerical evaluations.
\end{abstract}

Index Terms-Whitespaces, Dynamic Spectrum Access, TV White Space (TVWS), Cognitive Radio, Cellular Network, Unlicensed Spectrum, Channel Allocation Problem

\section{INTRODUCTION}

Cognitive radio (CR) systems allow dynamic access of secondary users (SU) to the licensed spectrum that is temporarily not used or underutilized by licensed (primary) users (PU). With the increasing usage of internet services by mobile users and the lack of sufficient spectrum, CR systems are becoming a popular solution for spectrum scarcity. For example the Federal Communication Commission (FCC) has opened unlicensed usage of $\mathrm{TV}$ band spectrum subject to specific regulations that prohibits harmful interference to TV broadcast services and other licensed users [1]-[3]. This possibility in TV band is mainly because of the switch-over from analog to digital TV transmission that was obligated by FCC for full power TV broadcasters in the United States. These opportunities have pushed the wireless industry to standardize CR operation in IEEE 802.22, 802.11af, etc. It is also expected that additional spectrum will be released for unlicensed use in the near future as result of the US National Broadband Plan [4].

A typical scenario for unlicensed operation requires $\mathrm{SU}$ to first detect the presence of unoccupied channels via spectrum sensing or by contacting a database administrator (DBA) to receive a list of available channels ${ }^{1}$. Every DBA models the protection region (or the so-called service contour) for each primary user based on FCC's protection rules and determines the list of available channels at each location/time. This framework for determining channel availability has two major effects. First, the computational burden of channel detection and incumbent protection is removed from secondary devices. Second, the DBA serves as an aggregation point for information about location and other technical specification of secondary devices, which can be utilized for possible centralized management solution (channel/power assignment) to secondary devices. This feature of TVWS networks (and possibly future WS spectrum) is unlike regular CR-based networks where devices are usually connected in adhoc mode without a centralized network controller/planner. This limitation eliminates the possibility of managing inter-network interferences that arise when independent networks utilize the shared spectrum, similar to the current situation of Wi-Fi networks that limits performance in dense urban areas [5]. Issues surrounding secondary network planning in TVWS is not well studied in the literature. Current FCC regulation for TVWS band are fully focused on protecting primary services [3] and does not consider interference management among TV based devices. This will eventually result in high interference floor for secondary devices and network throughput will degrade.

In this work, we focus on cellular secondary network design that operate on TVWS spectrum; however, the model and techniques apply to any WS scenario. Our model first and foremost accounts for FCC's protection contours of the primary sources. Second, the quality of each available WS channel is location dependent, due to varying interference levels from primary transmitters. Third, transmit power allowed on secondary TV band devices is also location dependent because of FCC's adjacent channel rules. Therefore, traditional techniques for resource allocation in cellular networks cannot be utilized and new approaches are needed.

The rest of the paper is organized as follows. Section II introduces secondary network architecture while coexisting with primary users. Section III reviews previous related work in this area. Channel allocation problem in secondary cellular networks is defined in section IV and various solutions are discussed in section V. Numerical results are provided in VI

\footnotetext{
${ }^{1} \mathrm{FCC}$ has adopted a centralized approach to secondary TVWS use by authorizing a group of DBAs to provide spectrum availability information.
} 
and finally section VII concludes the paper.

\section{Secondary Network Architecture}

Cellular communication systems are based on the notion of frequency reuse which allows a channel to be spatially reused by different users, as long as the co-channel interference is within acceptable bounds. However, for TVWS applications, the cellular layout of the secondary cells is further restricted by the primary protection regions, as shown in Fig. 1. This figure presents TV towers as an irregular primary network, where each primary cell corresponds to the coverage area (or service contour [3]) of the associated tower. $r_{i}$ denotes the maximum distance at which the received TV signal is above the detection threshold and the channel is considered busy [2] with an additional distance for protecting primary receivers on the edge of the service contour. Therefore, primary cell sizes depend on transmitter power/height, receiver sensitivity, terrain height profile and other physical layer parameters (details of calculation of primary user's protection area for TVWS is provided in [6]). It is clearly highlighted in this figure that service contour for TV broadcasters is often irregular and $r_{i}(\theta)$ significantly depends on $\theta$. The three protection regions in this example are obtained from [7]. This irregularity is mainly because of variations in terrain height along different directions (both average and variance) and the use of directional antenna by the transmitter.

Secondary networks can only be located outside of these protected contours subject to further FCC limitations imposed by need for protection of primary receivers. Secondary cells are usually much smaller in size compared to primary cells because of the lower transmission power and antenna height, Fig.1. The location dependency of secondary cells can be observed in this figure. Suppose primary transmitters in different colors are utilizing different channels. The area outside all protection regions can access the same set of available channels. However, secondary cells inside primary's protection region (shown as dotted circles) are possible as long as channel separation and power limitation is met by the secondary user. Therefore, these cells will receive a reduced set of available channels and those cells that are inside the overlapping section of protected regions receive an even shorter list. In practice, the number of protected entities are so large and diverse that essentially no secondary cell is completely outside all protection regions and list of channels are highly varying. As a practical example, in a highly populated urban area such as downtown Los Angeles there are zero channels available while 40 miles away from down town 14 channels are available for secondary operation as shown in Fig. 2.

Furthermore, the quality of available channels changes from cell to cell. This is because primary transmitters are very high power $^{2}$ and although secondary cells are outside protection regions they are still prone to receiving high interference from primaries especially for cells that are close to service contours.

\footnotetext{
${ }^{2}$ For full power digital TV transmitters, the transmitted power can be as big as $1000 \mathrm{kWatt}$.
}

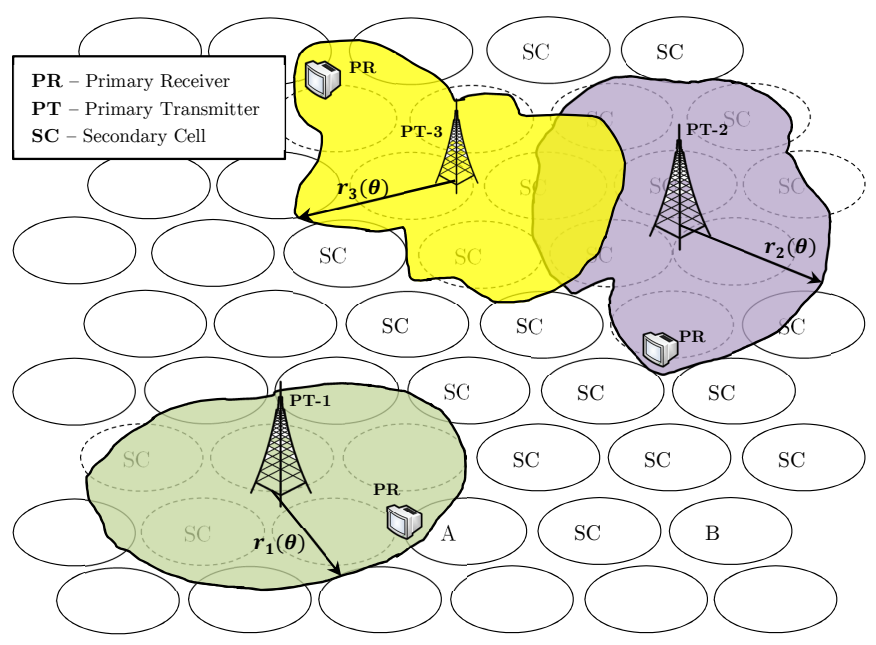

Fig. 1. Coexistence between primary and secondary cells in cellular networks based on TV white space. Primary cells are protection contours for TV broadcasters, defined based on transmission power, receiver sensitivity and other link-budget parameters [7].

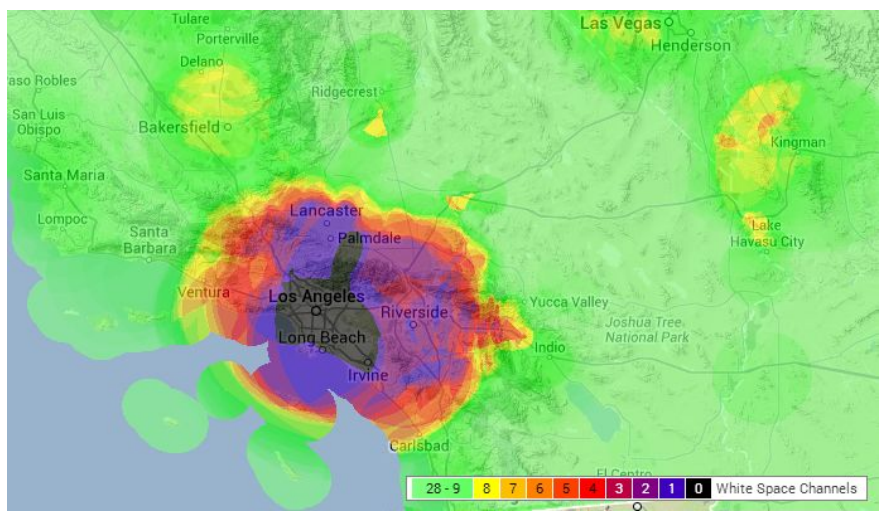

Fig. 2. Variability of available channels in a highly populated area (Los Angeles). Color map data are obtained from [8].

In Fig.1, cell A and B have the same set of available channels but significantly stronger interference affects A compared to B.

\section{RELATED WORKS}

Traditional channel allocation techniques for cellular networks [9] seek to optimize channel reuse factor while maintaining signal-to-interference plus noise (SINR) ratio for any link, by designing appropriate dynamic channel assignment algorithms [10]-[12]. Such channel assignment algorithms are modeled as a graph color/multi-coloring problem in which each node (base station) is colored according to the number of requests (user calls) in the associated cell. To achieve this, each node is weighted by the number of calls in the cell that includes currently ongoing calls and new requests [10]. Therefore, the objective target is to achieve the point where all cell calls are responded (assuming it is feasible). The major question of interest in this case is whether the graph is colorable or not instead of optimizing the total network throughput 
[9], [12], [13]. Network planning in a data network is beyond just responding to current requests. It should improve quality of service to the end user by maximizing overall throughput. Therefore the objective function to be optimized is network capacity (in its various forms and definitions) rather than the number of calls being covered.

What makes TVWS-based networks particularly interesting is the fact that the set of available channels changes from cell to cell and from device to device ${ }^{3}$. This is unlike regular cellular networks where there is no inherent differences among cells and potentially the same set of channels is available to every cell. In every primary-secondary paradigm, these variations in time/location/device-type complicate the channel assignment process and is not considered in the literature [13][20].

In [21], a distributed algorithm is considered for cognitive cellular networks using a game theory framework. However, major factors are overlooked in their problem formulations. Primary to secondary interference is not considered in calculating channel quality and the optimization metric is not the aggregate throughput. Only one channel is assigned to each AP which reduces the solution to a graph coloring instead of multi-coloring problem. Maximum power for each AP is decided solely based on primary limitations and not secondaryto-secondary interference and the method does not comply with FCC's vision of power selection [2], [3]. The protection contour for each primary user is modeled as a single point in their LP formulation which is technically wrong because the whole area should be protected.

In [22]-[24] greedy and IP-based algorithms are considered for formulating spectrum allocation problem. However, the aforementioned constraints for TVWS paradigm are not considered and the objective function is limited to some utility functions (such as total revenue for spectrum broker) rather than overall network throughput.

Our contribution in this paper is to formulate resource allocation problem in cellular networks from a data transfer perspective (rather than voice) and in a more generalized paradigm. We consider channel variation as well as different sources of interference (both secondary to secondary and primary to secondary) in the problem formulation in order to optimize overall network throughput.

\section{Channel Allocation in Secondary Cellular NETWORKS}

An interesting question for a network planner in TV white space spectrum is 'how to assign available channels to different users?' In regular cellular networks, repetitive patterns are used in which every channel is periodically (in space) assigned to nodes with specific distances. For example in a fully developed hexagonal structure, Fig. 3, channel assignment is defined by two shift parameters $i, j$. The resulting frequency reuse parameter $K=i^{2}+i j+j^{2}$ determines the

\footnotetext{
${ }^{3} \mathrm{FCC}$ defines different TVBD types (fixed versus portable) and regulations are different for each type. Hence, the set of available channels depends on the device type as well as other parameters.
}

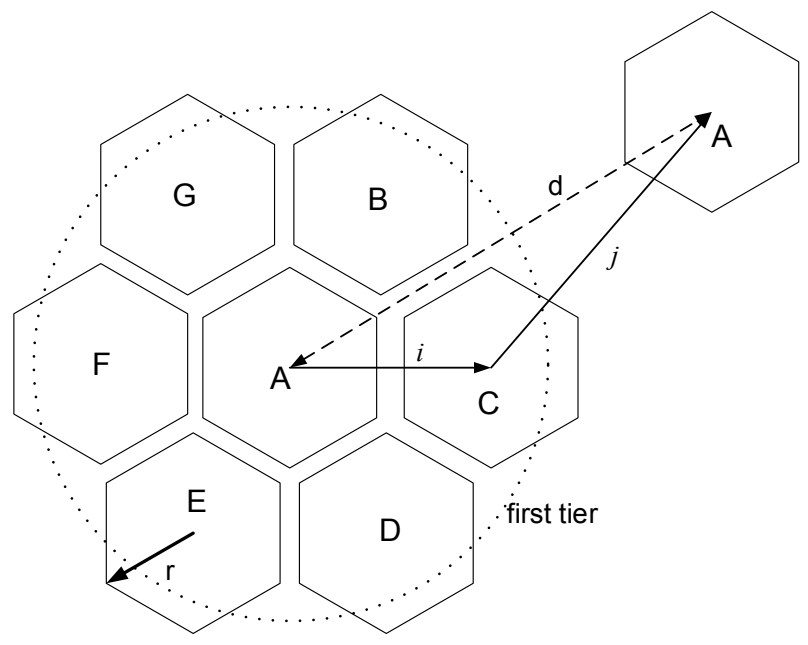

Fig. 3. Spatial frequency reuse in a fully developed hexagonal cellular network. Cells with similar letter share the same channel. No channel sharing exists among the first tier neighbors. Shift parameters $i, j$ defines how channel repeats in space.

frequency-reuse rate of each channel across the network [25]. In contrast, the same method can be used in TVWS only for those channels that are available in every cell (which are very few). The availability of channels is significantly a function of location. Furthermore, channels are shared with primary users, here TV broadcasters, which is a different network with a variant requirement that makes network planning nonhomogenous. The quality of each channel (in this context defined as signal to noise and interference ratio) depends on primary-to-secondary interference and is significantly channel and location dependent [6]. Therefore, any optimal channel allocation mechanism must consider these irregularities in the optimization process.

Let's define $\mathcal{C}$ as the set of all permissible white space channels [3]. Our focus is toward TV white space channels and therefore $\mathcal{C}=\{2,3, \ldots, 36,38, \ldots, 51\}$ with each channel representing $6 \mathrm{MHz}$ bandwidth in V/UHF band (based on USA standard [26]). For every cell $A_{i}, \Upsilon\left(A_{i}\right) \subseteq \mathcal{C}$ is the set of all available channels that must be determined according to incumbents protections and FCC regulations. A minimum of one channel must be allocated to each cell (with the assumption that $\Upsilon\left(A_{i}\right) \neq \emptyset$, there is at least one available channel in that cell). For each available channel $c \in \Upsilon\left(A_{i}\right)$, we specify a quality factor $\gamma_{i, P}(c)$ as the level of interference from primary transmitter. This parameter includes co-channel and adjacent channel pollution from all licensed users in channel $c$.

Since more than one channel can be assigned to each cell, the overall network throughput depends on how many channels are utilized in each cell without severe mutual interference. Therefore an optimizing algorithm toward maximizing the network throughput (either average or worst-case user throughput) can be set differently according to the level of details involved. Here, we consider two problem formulations, one for 
maximizing the total number of channels assigned to the entire network and another for maximizing total Shannon capacity of the network.

Problem definition 1: For a set of $N$ cells $\left\{A_{0}, A_{1}, \ldots, A_{N-1}\right.$ \} , with channel set availability of $\left\{\Upsilon\left(A_{0}\right), \ldots, \Upsilon\left(A_{N-1}\right)\right\}$, a channel selection function $f: \Upsilon\left(A_{i}\right) \rightarrow C_{i} \subset \Upsilon\left(A_{i}\right)$ is desired that assigns to each cell a subset of available channels $C_{i} \subset \Upsilon\left(A_{i}\right)$ so as to maximize $f_{\text {opt }}$ :

$$
f_{\text {opt }}=\arg \max _{f} \sum_{i=0}^{N-1}\left|C_{i}\right|
$$

subject to:

$$
\begin{aligned}
& C_{i} \subset \Upsilon\left(A_{i}\right), \forall i \in[0, \ldots, N-1] \\
& C_{i} \neq \emptyset \\
& \sum_{j=0}^{N-1} \gamma_{i, j}(c)+\gamma_{i, P}(c)+\sigma_{n}^{2} \leq \gamma_{t}, \forall c \in C_{i}
\end{aligned}
$$

where $\gamma_{i . j}(c)$ is the mutual interference between cell $i$ and $j$ on channel $c, \sigma_{n}^{2}$ is noise power and $\gamma_{t}$ is the maximum acceptable noise and interference threshold (In terms of transmission power $P$ and desired SNR, $\left.\gamma_{t}=\frac{P}{S N R}\right)$. The last inequality condition guarantees that channel noise floor is below a certain level and therefore an acceptable performance is achievable on the shared channels. If $c_{i}$ is not shared by cell $i$ and $j$ then $\gamma_{i . j}\left(c_{i}\right)=0$. This formulation maximizes the number of channels used by the cells but does not necessarily optimize throughput. This is because mutual interference is kept below a certain level rather than being optimized (minimized). The following more sophisticated formulation for this problem considers this issue.

Problem definition 2: For a set of $N$ cells, $\left\{A_{0}, A_{1}, \ldots\right.$, $\left.A_{N-1}\right\}$, with channel set availability of $\left\{\Upsilon\left(A_{0}\right), \ldots, \Upsilon\left(A_{N-1}\right)\right.$ \}, a channel selection function $f: \Upsilon\left(A_{i}\right) \rightarrow C_{i} \subset \Upsilon\left(A_{i}\right)$ is desired that assigns to each cell a subset of available channels $C_{i} \subset \Upsilon\left(A_{i}\right)$ as following:

$$
\begin{aligned}
& f_{\text {opt }}=\arg \max _{f} \sum_{i=0}^{N-1} \sum_{c \in C_{i}} \log _{2}(1+S I N R(c)) \\
& S I N R(c)=\frac{P}{\sum_{j=0}^{N-1} \gamma_{i, j}(c)+\gamma_{i, P}(c)+\sigma_{n}^{2}}
\end{aligned}
$$

subject to:

$$
\begin{aligned}
& C_{i} \subset \Upsilon\left(A_{i}\right), \forall i \in[0, \ldots, N-1] \\
& C_{i} \neq \emptyset
\end{aligned}
$$

Here, Shannon equation is used for the throughput of each channel utilized in each cell. Therefore, the answer to this optimization problem is optimal in the sense of overall secondary network's throughput.

\section{Solutions to Channel Allocation Problem}

Finding a solution to channel assignment is generally very challenging and it highly depends on the level of details involved. Here, problem definition 1 is focused toward maximizing the number of assigned channels that is a discrete function with continuous constraints. On the other hand, problem definition 2 is optimizing a continuous function with continuous constraints. While both problems are discrete optimization, the latter is significantly more complicated that the former. In following sections, we explore possible solutions for both problems.

\section{A. Suboptimal Greedy Solution for Problem 1}

One sub-optimal solution for problem 1 is possible by considering this fact that in cellular systems, the strongest interferer is the first tier in a hexagonal structure as shown in Fig. 3. Because of this, any frequency reuse pattern avoids using the same channel in neighboring cells. Therefore, as a mechanism to ensure interference rejection, we force the solution to avoid reusing in neighboring cells (can be generalized to prevention against second and third tiers) while the total number of channels in all cells is maximized.

In order to generalize this idea for our problem, we modify the hard decision mechanism based on adjacency to a soft decision process that eliminates channels from neighbors based on comparing overall calculated interference with $\gamma_{t}$. Let's model the network as a graph $G=(V, E)$ where $V$, the set of vertices, represents the cell centers (base stations) and $E$, the set of edges, describes cell adjacency. Therefore, if $e=\left(v_{1}, v_{2}\right) \in E$ then $v_{1}$ and $v_{2}$ are two neighboring cells. This can be generalized to second/third-tier cells if cells are small and interference beyond the first neighbors are considerable. For every edge $v_{i} \in V$, the set of available channels is $\Upsilon\left(v_{i}\right) \subset \mathcal{C}$ as shown in Fig. 4. The set of assigned channels to each vertex $v_{i}$ is $C\left(v_{i}\right)$.

Here we explore a greedy algorithm that seeks to assign non-shared channels first and then divide the remaining set of channels evenly between interfering cells. The algorithm, shown in Algorithm 1, starts with a vertex that has minimum number of assigned channels and minimum number of available channels. Every possible channel is explored on this node to find the one that results in minimum number of interfered nodes. A node $v$ is considered interfered as the result of a channel assignment if one of the following happens:

- Total interference level in $v$ crosses the threshold $\gamma_{t}$

- Assigning this channel to node $v$ results in another currently assigned node to cross the threshold

The channel $c_{\text {min }}$ that causes minimum number of interfered nodes is assigned to current vertex and is removed from the set of available channels. The list of available channels for all neighboring nodes is updated after this assignment according to two condition above. This process is repeated until there is no available channel in all the vertices.

Greedy algorithm guarantees every assigned channel $c \in C_{i}$ has a total interference level less than threshold $\gamma_{t}$. However, it does not guarantee the condition of $C_{i} \neq \emptyset$ is met. The algorithm reaches most vulnerable nodes first to minimize the probability of having $C_{i}=\emptyset$ but the final results depend on the distribution of the available channels among nodes and 


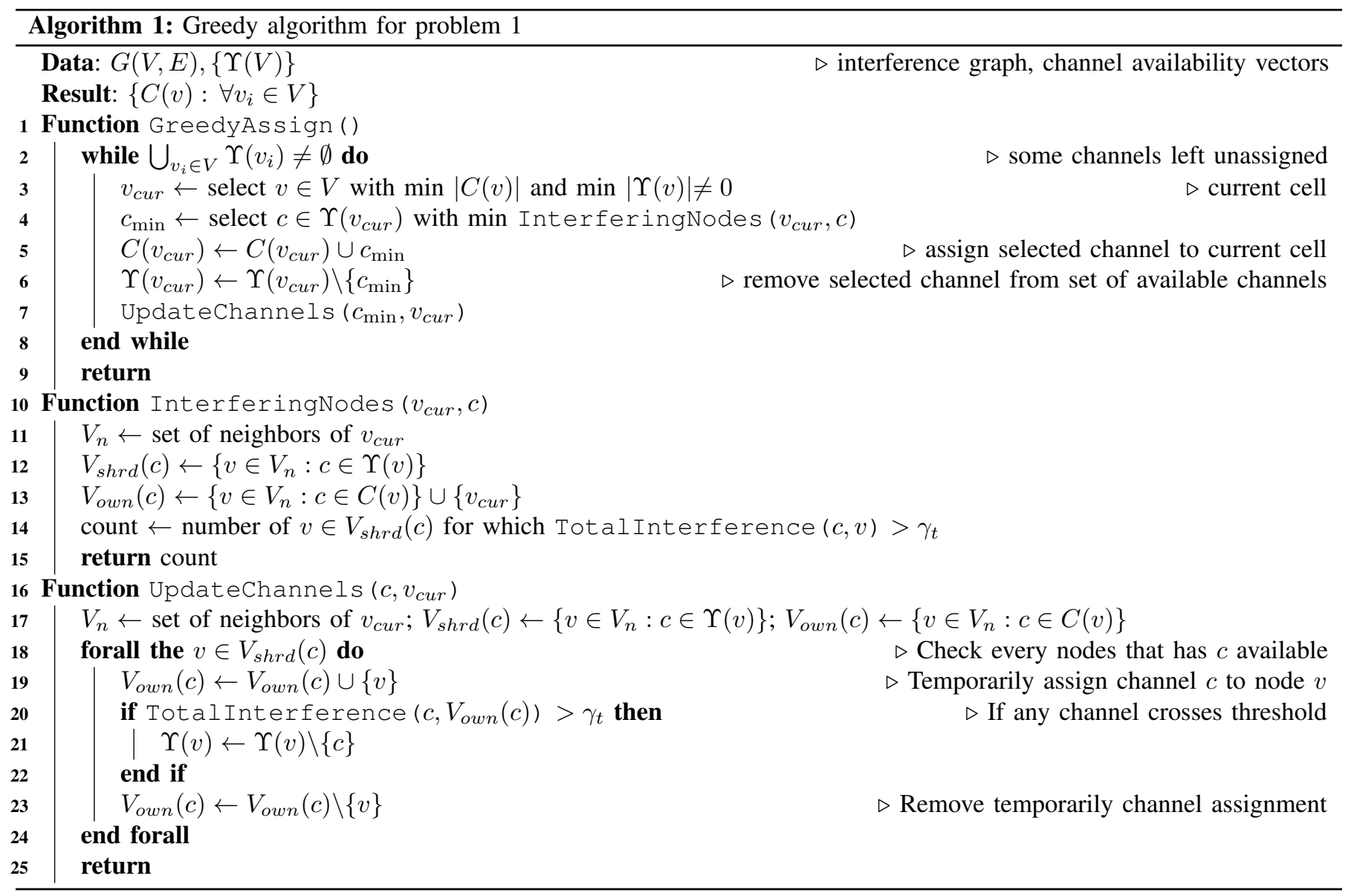

the value of $\gamma_{t}$. There are very rare cases for large values of $\gamma_{t}$ that there is a solution which meets the constraints but this algorithm barely misses that. However, all these cases are for large and non-optimal values of $\gamma_{t}$ as we will see in the numerical section results. For a wide range of $\gamma_{t}$ that includes the optimal point, this algorithm also satisfies this criteria.

\section{B. Integer Programming formulation for problem 1}

In this section we formulate the channel assignment problem as an integer linear program (ILP). The reason for this translation is the availability of standard tools and libraries for solving ILP. Classic channel assignment problems are usually modeled as a graph multi-coloring (GMC) problem [27]. However, there are major differences between our resource allocation problem and GMC. In a typical GMC setup, the objective function is to minimize the number of required channels (colors) to cover a specific interference graph such that each node receives the minimum required channels. Therefore, there is a homogeneity in GMC that does not exist in our problem. Here, the set of channels that are available at each node is forced by the scenario and changes from node to node. There is a quality factor assigned to each channel that originates from primary to secondary interference and the objective function is to maximize the total number of assigned channels in the network.
For each node $v_{i} \in V(i=0, \ldots, N-1)$ let $\mathcal{A}_{i}^{|\mathcal{C}| \times 1}$ be a binary vector of length $|\mathcal{C}|$ (total number of channels) that defines availability/unavailability of each channel at node $v_{i}$. Also, let $\mathcal{L}_{i}^{|\mathcal{C}| \times 1}$ be the binary vector that shows what channels are currently assigned to node $v_{i}$. We define $\mathcal{A}$ and $\mathcal{L}$ as the concatenation of all $\mathcal{A}_{i}$ and $\mathcal{L}_{i}$ vectors, respectively:

$$
\mathcal{A}=\left[\begin{array}{c}
\mathcal{A}_{0} \\
\mathcal{A}_{1} \\
\ldots \\
\mathcal{A}_{N-1}
\end{array}\right], \mathcal{L}=\left[\begin{array}{c}
\mathcal{L}_{0} \\
\mathcal{L}_{1} \\
\ldots \\
\mathcal{L}_{N-1}
\end{array}\right]
$$

The optimization problem is to find the optimum $\mathcal{L}$ that has maximum weight and yet satisfies the constraints. All the constraints in problem 1 are now defined in terms of $\mathcal{L}$ and $\mathcal{A}$. The availability constraint is specified as $\mathcal{L}^{T} \cdot \mathcal{A}^{c}=0$ where $(.)^{T}$ is matrix transpose operation and $\mathcal{A}^{c}$ is binary complement of $\mathcal{A}$. The minimum number of assigned channels to each node (that must be non-zero $C_{i} \neq \emptyset$ ) is defined in terms of $\mathcal{L}$ as:

$$
\sum_{j=0}^{|\mathcal{C}|-1} \mathcal{L}(|\mathcal{C}| i+j)>0, \quad i=0,1, \ldots, N-1
$$

In order to formulate mutual interference condition, let's translate the general from defined by (2) in our ILP problem. This constraint should be specified for every $v_{i} \in V$ and for 


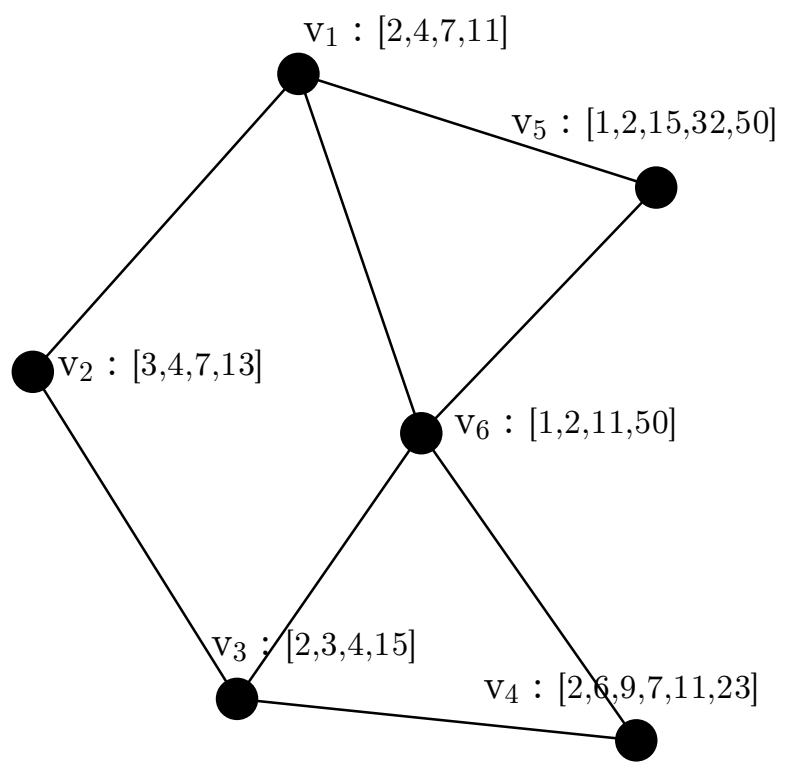

Fig. 4. Graph based model for cellular networks in TVWS spectrum. Cells are represented by vertices, along with their corresponding set of channels and interfering cells are connected by edges.

every permissible channel $c_{k} \in \Upsilon\left(v_{i}\right)$. We assume the cross interference is known for every pair of $\left(v_{i}, v_{j}\right)$ as $\gamma_{i, j}\left(c_{k}\right)$. For simplicity of notation, we assume $\gamma_{i, i}(c)=0$. Thus, for every $v_{i} \in V$ and for every channel $c_{k} \in \Upsilon\left(v_{i}\right)$ we need an interference constraint as below:

$$
\begin{aligned}
& \sum_{j=0}^{N-1} \mathcal{L}(j|\mathcal{C}|+k) \gamma_{i, j}\left(c_{k}\right) \\
& \quad+\gamma_{i, P}\left(c_{k}\right)+\sigma_{n}^{2} \leq[1-\mathcal{L}(i|\mathcal{C}|+k)] \Lambda+\gamma_{t}
\end{aligned}
$$

where $\Lambda \gg \gamma_{t}$ is a large constant number that is used here to make sure interference constraint is applied only when $\mathcal{L}(i|\mathcal{C}|+k)=1$ (when channel $c_{k}$ is actually assigned to $v_{i}$ ).

The ILP that finds the optimum solution to problem definition 1 is:

$$
\max _{\mathcal{L}} \mathbf{1}^{T} \cdot \mathcal{L}
$$

Subject to:

$$
\begin{aligned}
& \mathcal{L}^{T} \cdot \mathcal{A}^{c}=0 \\
& \sum_{j=0}^{|\mathcal{C}|-1} \mathcal{L}(|\mathcal{C}| i+j)>0, \quad i=0,1, \ldots, N-1 \\
& \sum_{j=0}^{N-1} \mathcal{L}(j|\mathcal{C}|+k) \gamma_{i, j}\left(c_{k}\right)+\gamma_{i, P}\left(c_{k}\right)+\sigma_{n}^{2} \leq \\
& \gamma_{t}+[1-\mathcal{L}(i|\mathcal{C}|+k)] \Lambda
\end{aligned}
$$

The answer to this ILP, if exists, is the optimal solution for problem definition 1 in (2). Compared to greedy algorithm, this formulation finds the best answer that maximizes the desired metric (number of assigned channels) and satisfies all required constraints. Clearly, trade off here is significantly higher complexity than the greedy method. However, the set of parameters here are sufficiently general that other restrictions can easily be applied to (9).

\section{Suboptimal greedy solution for problem 2}

A heuristic approach for finding a solution to problem 2 must consider maximizing the total throughput in (3) at every iteration. A greedy algorithm is shown in Algorithm 2. This procedure again starts with a node $v$ that has minimum number of assigned channels $|C(v)|$ and minimum number of available channels $|\Upsilon(v)|$. This is essential for our fairness constraint of $C_{i} \neq \emptyset$.

The procedure for finding best available channel $c_{\max }$ for current node $v$ is through estimating total achievable throughput (heuristically) for every available channel and choosing the one with maximum value. Estimation of total throughput is non-trivial. Our heuristic method (function BestChannel in Algorithm 2) starts by adding channel $c_{i}$ (from set of available channels) to $v$ and evaluate total throughput (considering mutual interferences). Afterward, as long as the value of total throughput is increasing, it keeps assigning channel $c_{i}$ to other nodes. This operation stops when total throughput is no longer increasing. This procedure is repeated for every channel and the maximum value of total throughput determines $c_{\max }$.

The neighbor removal process (function RemoveNeighbors in the Algorithm) is based on hypothetically assigning the channel to all eligible nodes and evaluating total throughput. If total throughput decreases as the result of such a channel assignment, the channel must be removed from set of available channels.

\section{Nonlinear IP formulation for problem 2}

Since the objective function in (3) is non-linear, regular integer programming formulation cannot be used here. Finding a solution to non-linear IP problems are generally NP-hard and therefore computationally they are not efficient. However, the result obtained from this formulation is optimal. This definition is mostly used as a benchmark against heuristicbased methods such as greedy algorithm in the previous section, to evaluate how close those results are to the optimal answer. The non-linear IP in this case is defined as below:

$$
\max _{\mathcal{L}} f_{o b j}(\mathcal{L})
$$

Subject to:

$$
\begin{aligned}
& \mathcal{L}^{T} \cdot \mathcal{A}^{c}=0 \\
& \sum_{j=0}^{|\mathcal{C}|-1} \mathcal{L}(|\mathcal{C}| i+j)>0, \quad i=0,1, \ldots, N-1
\end{aligned}
$$

where the objective function $f_{\text {obj }}(\mathcal{L})$ is defined based on total throughput in (3). Therefore, in terms of channel assignment 


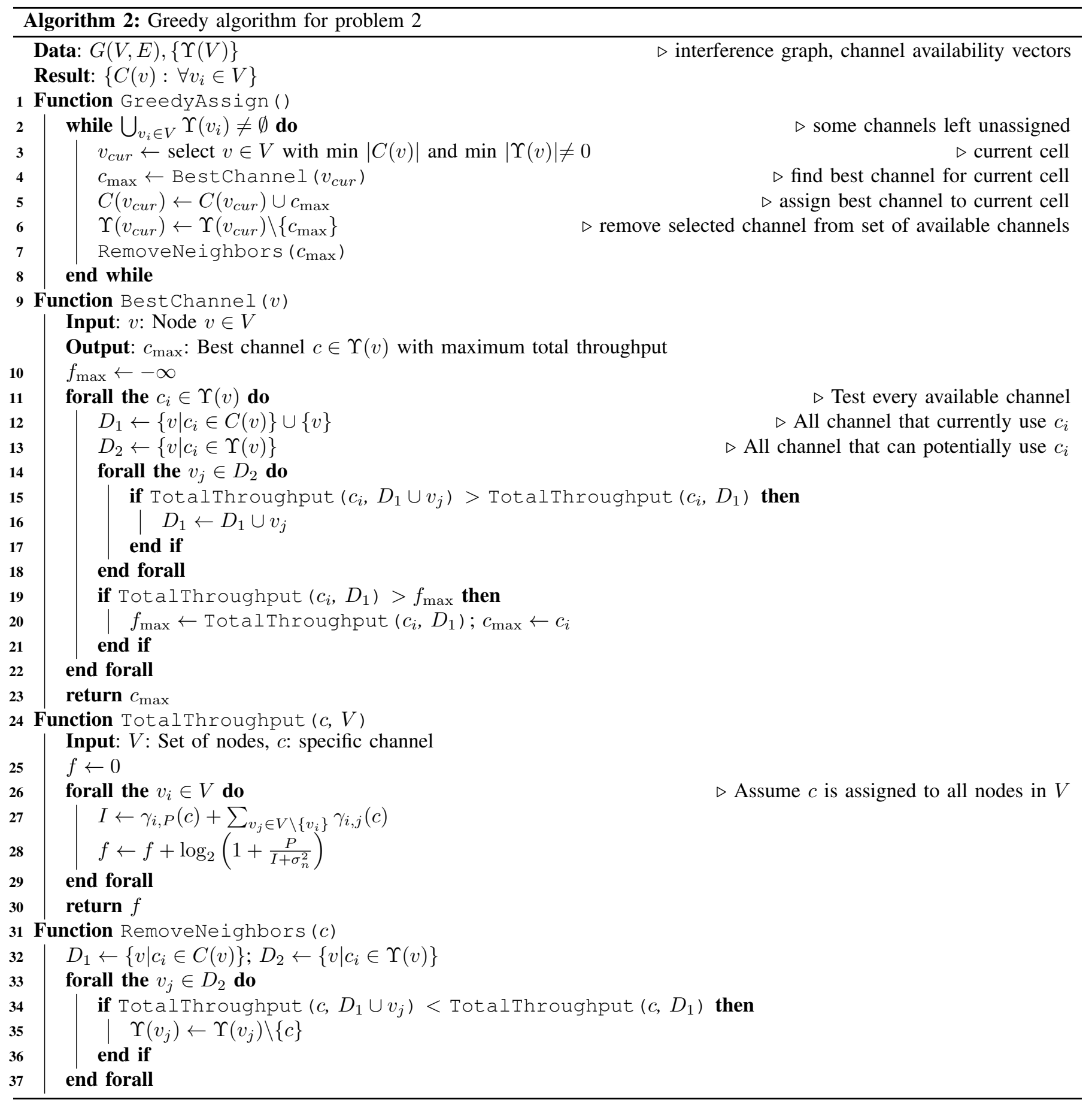

vector $\mathcal{L}$, it is defined as:

$$
\begin{aligned}
& f_{o b j}(\mathcal{L})=B^{1 \times N|\mathcal{C}|} \cdot \mathcal{L} \\
& B(i|\mathcal{C}|+k)= \\
& \quad \log _{2}\left(\frac{P}{\sum_{j=0}^{N-1} \mathcal{L}(j|\mathcal{C}|+k) \gamma_{i, j}\left(c_{k}\right)+\gamma_{i, P}\left(c_{k}\right)+\sigma_{n}^{2}}+1\right) \\
& i, k=0, \ldots, N-1
\end{aligned}
$$

\section{Numerical Results}

In this section, numerical results are provided for a typical network graph to compare introduced algorithms. Simulation parameters are provided in Table I. There are 25 nodes in a grid topology, Fig. 5. All secondary users transmit with unit power. Mutual interference between nodes is determined in terms of cross distance and typical path-loss functions $\gamma_{i, j} \propto \frac{P}{d_{i}^{2}}$. There are a total of 10 available channels that are randomly available at each node (uniform distribution in range of 3 to 5 


\begin{tabular}{|c|c|c|c|c|}
\hline$(((-i)))$ & $(((\cdot))))$ & $(((\cdot i)))$ & $((\cdot, \cdot))$ & $((\mathrm{k}, 1)))$ \\
\hline$(((0))))$ & $\left.v_{\left(v_{i}\right)}\left(\left(v_{i}\right)\right)\right)$ & $((0,1))$ & $\left(\left(p_{0}\right)\right)$ & $(((\cdot, p)))$ \\
\hline$((t \cdot 0)))$ & $\left(\left(\mathrm{l}_{\mathbf{2}}(1)\right)\right.$ & $d_{i, j}\left(\left(\left(_{i}\right)\right)\right)$ & $((\cdot, \cdot))$ & $\left(\left(\right.\right.$ (ipl) $\left.\left._{\mathrm{i}}\right)\right)$ \\
\hline$(((\cdot)))$ & $\left(\left(\right.\right.$ (i. $\left.\left._{\mathbf{i}}\right)\right)$ & 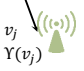 & $\left(\left({ }^{\prime}(p)\right)\right)$ & $(((\cdot)))$, \\
\hline$(((\vec{p})))$ & $\left.\left(\mathrm{g}_{\mathrm{R}}\right){ }^{2}\right)$ & $\left(\left(\left(a_{i}\right)\right)\right)$ & 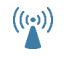 & $\left(\left(i_{i}, 1\right)\right)$ \\
\hline
\end{tabular}

Fig. 5. Network topology with multiple nodes in DSA scenario. Each node has a certain set of available channels $\Upsilon\left(v_{i}\right) \in\{1,2, \ldots, 10\}$.

for each node). The quality of each channel, defined in terms of primary to secondary interference level is also randomized for each channel uniformly.

TABLE I

SIMULATION PARAMETERS

\begin{tabular}{|c|c|}
\hline \hline Parameter & Value \\
\hline Number of nodes $|V|$ & 25 \\
Number of channels $|\mathcal{C}|$ & 10 \\
Available channels $\Upsilon(v)$ & $\sim U[3,5]$ \\
Primary Interference $\gamma_{i, P}()$. & $\sim U[0.0,0.1]$ \\
Secondary User's Power & 1.0 \\
Mutual Interference $\gamma_{i, j}$ & $\propto \frac{P}{d_{i, j}^{2}}$ \\
Noise Power $\sigma_{n}^{2}$ & 0.01 \\
\hline \hline
\end{tabular}

Each simulation scenario in the following two sections is repeated multiple times with different seeds for random generator and the results are averaged. The seed values however are fixed across different algorithm to provide a reasonable comparison. We keep a fixed seed for generating the number of available channels at each node but a variable seed is used for generating the actual channel numbers and channel quality factor. Therefore, the total number of available channels are fixed in all cases but the interference pattern is different.

The two factors of interest are the total number of channels that are assigned to nodes and the total throughput of the network $(\mathrm{bps} / \mathrm{Hz})$. We normalize both metrics by the total number of available channels for easier comparison and to remove dependency of the results on this factor.

\section{A. Comparing greedy algorithm with ILP in problem 1}

An important factor in problem 1 is parameter $\gamma_{t}$. This defines how conservative the algorithm is in terms of accepting external interference in an assigned channel. Increasing $\gamma_{t}$ will decrease the frequency reuse factor for the network and forces reused channels to be further away. Figure 6 shows the total number of assigned channels versus $S N R=\frac{P}{\gamma_{t}}$ for both greedy algorithm and ILP in problem 1. Decreasing SNR (increasing $\gamma_{t}$ ) will increase the total number of channels

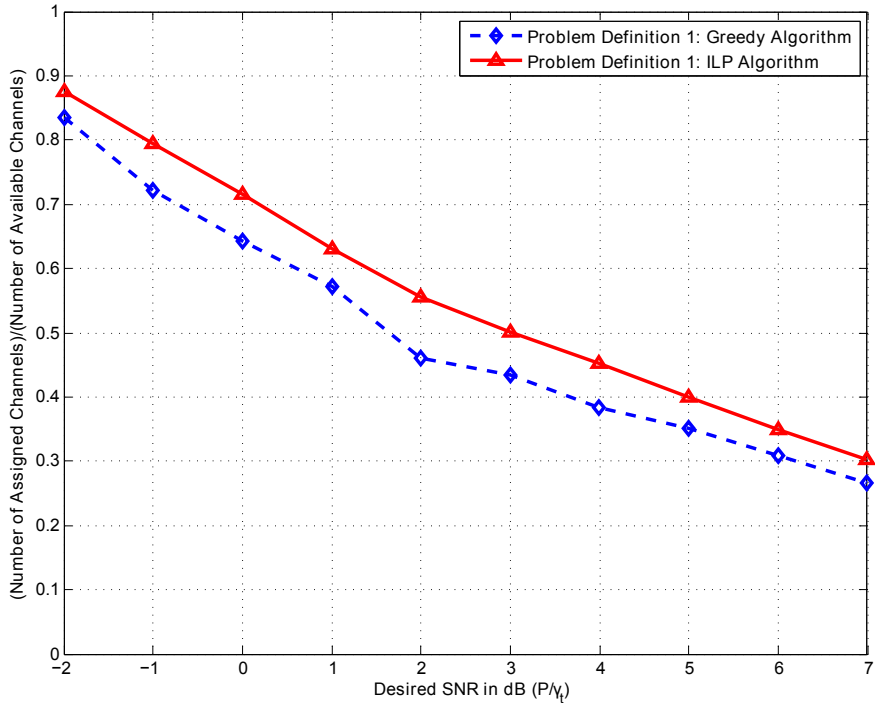

Fig. 6. Number of assigned channels normalized to total number of available channels versus $\mathrm{SNR}=\frac{P}{\gamma_{t}}$ in (2) for first problem formulation.

in both algorithms because higher level of interference is accepted. The two algorithms present the same behavior and their performance is relatively close.

Figure 7 shows the normalized total throughput of the network in terms of bps/Hz/(Available Channel) for both methods. The total throughput is calculated by considering total interference at each node as sum of mutual interference, primary to secondary interference and noise power for every assigned channel and utilizing Shannon capacity equation. The trade-off between throughput and $\gamma_{t}$ can be seen in this figure. For smaller values of $\gamma_{t}$, throughput decreases because the fewer number of channels becomes dominant. For larger values of $\gamma_{t}$ it also decreases because excessive interference becomes dominant. While both methods present a similar behavior overall, the trade-off is more visible in ILP. This is because in ILP problem, the algorithm forces one channel per node and the target function is to maximize the total channels. This can result in multiple channels at one node while another node has only one channels assigned. On the other hand, greedy algorithm provides a more homogenous distribution of channels among nodes because always the node with minimum $|C(v)|$ is selected. Therefore, this algorithm shows less sensitivity to threshold value.

\section{B. Comparing problem 2 with problem 1}

With the lack of standard tools for solving nonlinear IP problems, our work in providing the most optimal answer through second problem definition is an ongoing effort for future works. However, in this section we can compare the results of greedy algorithm for problem 2 versus one. Problem definition 2 is based on maximizing total throughput without utilizing hard decision threshold for channel assignment. Therefore, the choice of $\gamma_{t}$ does not affect the result. Hence, we compare this result with best results obtained from problem 1 through a search in $\gamma_{t}$. Table II compares the results for 


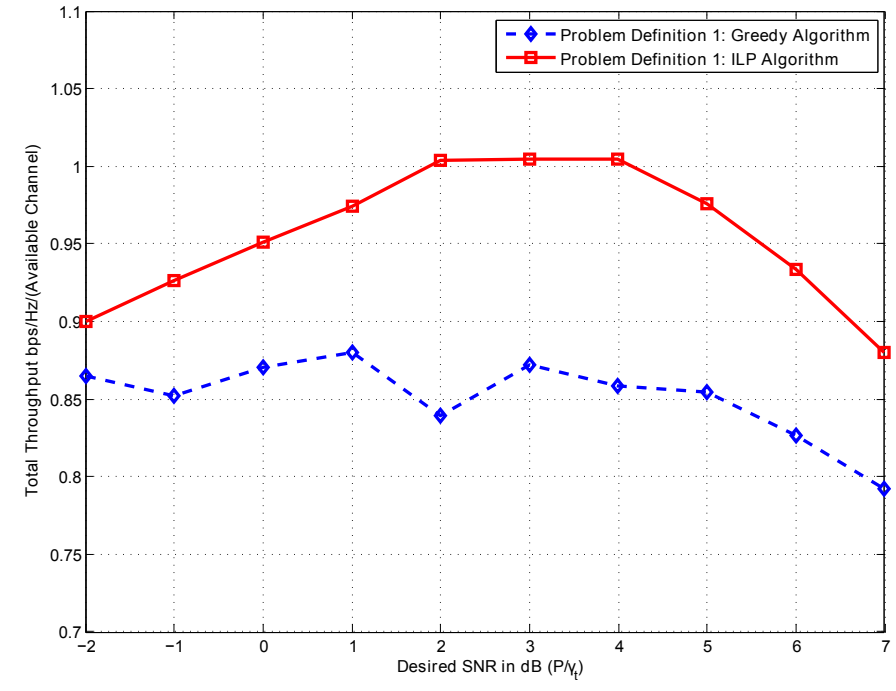

Fig. 7. Total throughput across the network normalized by total number of available channel versus SNR $=\frac{P}{\gamma_{t}}$ in (2) for first problem formulation. Mutual interference between nodes, primary to secondary interference and noise variance is considered in calculation of throughput.

TABLE II

COMPARING SIMULATION RESULTS FOR PROBLEM 2 VERSUS PROBLEM 1

\begin{tabular}{|c|c|c|}
\hline Method & Norm. No. Assigned Channels & Norm. Throughput \\
\hline $\begin{array}{l}\text { Greedy Prob. 2 } \\
\text { with IQP }\end{array}$ & 0.534 & 0.976 \\
\hline $\begin{array}{l}\text { Greedy Prob. 2 } \\
\text { with Heuristic }\end{array}$ & 0.546 & 0.973 \\
\hline ILP Prob. 1 & 0.5 & 1.005 \\
\hline Greedy Prob. 1 & 0.57 & 0.88 \\
\hline \hline
\end{tabular}

both definitions. In addition to greedy method in Algorithm 2 , we simulated a different greedy method that runs an integer quadratic programming (IQP) for minimizing total interference in every iteration inside method BestChannels. The results show slightly better performance than the previous heuristic based method. By comparing the results in this table, it is inferred that ILP presents the best results and Greedy for problem 1 which has the worst performance is only $12 \%$ below ILP. Greedy for problem 2 is very close to ILP, only $2.8 \%$ below.

Considering both performance and computational requirements, greedy algorithm for problem 2 has the best performance. This is because of multiple reasons. First, problem formulation 2 does not depend on threshold and automatically finds the optimal point. Thus, no exhaustive search is needed. Second, greedy algorithm is significantly faster in every iteration. Third, the complexity of greedy algorithm is polynomial in number of nodes while ILP is exponential. Therefore, greedy method is better scalable. Finally, the overall result of greedy is sufficiently close to ILP.

\section{CONCLUSION}

In this paper, we explored the problem of resource allocation in TVWS based networks with a focus on cellular networks. Major issues in TVWS were addressed including variability of available channels w.r.t. location as well as quality of each channel. Two problem definitions were presented for allocating channels to cells with different objective functions and constraints. First problem formulation, based on maximization of total number of channels, proved to be an integer linear programming problem for which standard tools are available. Second problem formulation was based on maximization of total Shannon throughput which resulted in a non-linear IP problem. Greedy algorithms were provided for both definitions.

Numerical results showed a trade off between total throughput and $\gamma_{t}$ for problem 1 and that careful choice of $\gamma_{t}$ is needed to obtain optimum results. In practice, exhaustive/binary search techniques are required to find optimum $\gamma_{t}$. By choosing the best value of $\gamma_{t}$, ILP showed the best results which was slightly better than greedy solution for problem 2 with the cost of enormously higher computation.

\section{REFERENCES}

[1] In the Matter of Unlicensed Operation in the TV Broadcast Bands: Second Report and Order and Memorandum Opinion and Order, FCC Std. 08-260, Nov. 2008.

[2] In the Matter of Unlicensed Operation in the TV Broadcast Bands: SECOND MEMORANDUM OPINION AND ORDER, FCC Std. 10-174, Sept. 2010.

[3] In the Matter of Unlicensed Operation in the TV Broadcast Bands: THIRD MEMORANDUM OPINION AND ORDER, FCC Std. 12-36, April 2012.

[4] "The national broadband plan: Connecting america," Federal Communications Commission, Tech. Rep., March 2010, http://www.broadband.gov/plan/.

[5] M. A. Ergin, K. Ramachandran, and M. Gruteser, "Understanding the effect of access point density on wireless lan performance," in Proceedings of the 13th annual ACM international conference on Mobile computing and networking, ser. MobiCom '07. New York, NY, USA: ACM, 2007, pp. 350-353. [Online]. Available: http://doi.acm.org/10.1145/1287853.1287902

[6] F. Hessar and S. Roy, "Capacity Considerations for Secondary Networks in TV White Space," University of Washington, Tech. Rep., 2012.

[7] Cloud Based Simulation Engine for TVWS. [Online]. Available: http://specobs.ee.washington.edu/

[8] Google Spectrum Database. [Online]. Available: https://www.google.com/get/spectrumdatabase/

[9] D. Cox and D. Reudink, "Dynamic Channel Assignment in TwoDimensional Large-Scale Mobile Radio Systems," The Bell Systems Technical Journal, vol. 51, no. 7, pp. 1611-1629, 1972.

[10] L. Narayanan, Handbook of wireless networks and mobile computing. New York, NY, USA: John Wiley \& Sons, Inc., 2002, ch. Channel assignment and graph multicoloring, pp. 71-94. [Online]. Available: http://dl.acm.org/citation.cfm?id=512321.512325

[11] W. Si, S. Selvakennedy, and A. Y. Zomaya, "An overview of Channel Assignment methods for multi-radio multi-channel wireless mesh networks," J. Parallel Distrib. Comput., vol. 70, no. 5, pp. 505-524, May 2010. [Online]. Available: http://dx.doi.org/10.1016/j.jpdc.2009.09.011

[12] I. Katzela and M. Naghshineh, "Channel assignment schemes for cellular mobile telecommunication systems: a comprehensive survey," Personal Communications, IEEE, vol. 3, no. 3, pp. 10-31, 1996.

[13] D. Martinez, A. Andrade, and A. Martinez, "Interference-aware dynamic channel allocation scheme for cellular networks," in Performance Evaluation of Computer and Telecommunication Systems (SPECTS), 2010 International Symposium on, 2010, pp. 295-300.

[14] A. Mishra, S. Banerjee, and W. Arbaugh, "Weighted coloring based channel assignment for WLANs," SIGMOBILE Mob. Comput. Commun. Rev., vol. 9, no. 3, pp. 19-31, Jul. 2005. [Online]. Available: http://doi.acm.org/10.1145/1094549.1094554

[15] C. Zhao and L. Gan, "Dynamic Channel Assignment for Large-Scale Cellular Networks Using Noisy Chaotic Neural Network," Neural Networks, IEEE Transactions on, vol. 22, no. 2, pp. 222-232, 2011. 
[16] Y. Tam, R. Benkoczi, H. Hassanein, and S. Akl, "Channel Assignment for Multihop Cellular Networks: Minimum Delay," Mobile Computing, IEEE Transactions on, vol. 9, no. 7, pp. 1022-1034, 2010.

[17] J. Nasreddine, A. Achtzehn, J. Riihijarvi, and P. Mahonen, "Enabling Secondary Access through Robust Primary User Channel Assignment," in Global Telecommunications Conference (GLOBECOM 2010), 2010 IEEE, 2010, pp. 1-5.

[18] V. Chandrasekhar and J. Andrews, "Spectrum allocation in tiered cellular networks," Communications, IEEE Transactions on, vol. 57, no. 10, pp. 3059-3068, 2009.

[19] K. Doppler, S. Redana, M. Wódczak, P. Rost, and R. Wichman, "Dynamic resource assignment and cooperative relaying in cellular networks: concept and performance assessment," EURASIP J. Wirel. Commun. Netw., vol. 2009, pp. 24:1-24:14, Jan. 2009. [Online]. Available: http://dx.doi.org/10.1155/2009/475281

[20] Q. Xin and J. Xiang, "Joint QoS-aware admission control, channel assignment, and power allocation for cognitive radio cellular networks," in Mobile Adhoc and Sensor Systems, 2009. MASS '09. IEEE 6th International Conference on, 2009, pp. 294-303.

[21] D. Li and J. Gross, "Distributed TV Spectrum Allocation for Cognitive Cellular Network under Game Theoretical Framework," in Proc. IEEE International Symposium on Dynamic Spectrum Access Networks DYSPAN'12, 2012, pp. 327-338.

[22] A. Subramanian, M. Al-Ayyoub, H. Gupta, S. Das, and M. Buddhikot, "Near-optimal dynamic spectrum allocation in cellular networks," in New Frontiers in Dynamic Spectrum Access Networks, 2008. DySPAN 2008. 3rd IEEE Symposium on, 2008, pp. 1-11.

[23] L. Cao, L. Yang, X. Zhou, Z. Zhang, and H. Zheng, "Optimus: Sinrdriven spectrum distribution via constraint transformation," in New Frontiers in Dynamic Spectrum, 2010 IEEE Symposium on, 2010, pp. $1-12$.

[24] S. Im and H. Lee, "Dynamic spectrum allocation based on binary integer programming under interference graph," in Personal Indoor and Mobile Radio Communications (PIMRC), 2012 IEEE 23rd International Symposium on, 2012, pp. 226-231.

[25] W. C. Y. Lee, Wireless and Cellular Telecommunication, 3rd ed. McGraw-Hill, 2005.

[26] IEEE P1631/D3 Draft Recommended Practice for Measurement of 8VSB Digital Television Mask Transmission Compliance for the USA, IEEE Broadcast Technology Society Std. P1631/D3, Feb. 2008

[27] M. Anuj and T. M. A., "A branch-and-price approach for graph multicoloring," Extending the Horizons: Advances in Computing, Optimization, and Decision Technologies, p. 1529, 2007. 